COMMUNICATIONS IN

ANALYSIS AND GEOMETRY

Volume 5, Number 3, 475-495, 1997

\title{
Realization theorems of geometric singularities for Hamilton-Jacobi equations
}

\author{
Shyuichi IZumiYa AND Georgios T. Kossioris
}

\section{0 . Introduction.}

In $([10],[11])$ we described the geometric framework for the study of generation and propagation of shock waves in $\mathbb{R}^{n}$ appearing in viscosity solutions of Hamilton-Jacobi equations

$$
\left\{\begin{array}{l}
\frac{\partial y}{\partial t}+H\left(t, x_{1}, \cdots, x_{n}, \frac{\partial y}{\partial x_{1}}, \cdots, \frac{\partial y}{\partial x_{n}}\right)=0 \\
y\left(0, x_{1}, \cdots, x_{n}\right)=\phi\left(x_{1}, \cdots, x_{n}\right)
\end{array}\right.
$$

where $H$ and $\phi$ are $C^{\infty}$-functions.

The geometric solution of $(\mathrm{P})$ has been defined in ([10],[11]) which is constructed by the method of characteristics. Although it is initially smooth there is in general a critical time beyond which characteristics cross. The geometric solution past the critical time is multi-valued, that is singularities appear. The purpose of the papers $([10],[11])$ is to give a correct geometrical setting for describing the shock waves of viscosity solutions (cf., [6],[7]). Existence and uniqueness of the solution of $(\mathrm{P})$ in the viscosity sense have been established in [6]. The viscosity solution is continuous and coincides with the smooth geometric solution until the first critical time. After the characteristic cross, the viscosity solution developes shock waves i.e., surfaces across which the gradient of the viscosity solution is discontinuous. The viscosity solution of $(\mathrm{P})$ in a neighbourhood of the first critical time has been constructed in [14] by selecting a continuous single-valued branch of the graph of the geometric solution. Our project is to continue this process and construct the viscosity solutions around each singularity of the geometric solution. So we need to classify the singularities of geometric solutions.

On the other hand, the notion of Legendrian unfoldings has been defined in [9] for the study of completely integrable first order differential equations. In $([10],[11])$ it has been shown that the geometric solution of $(P)$ is locally 
an one-parameter Legendrian unfolding and presented the classifications of singularities of generic Legendrian unfoldings. For our purpose we have to prove that one-parameter Legendrian unfoldings is the correct class which describes the singularities of geometric solutions. So the main results in this paper are the realization theorems for one-parameter Legendrian unfoldings as geometric solutions of a given Hamilton-Jacobi equation (cf., 'Theorems $3.2,3.3)$. We can also specify the point at where the generic bifurcations of geometric singularities occur.

Recently, there appeared several articles studying this subject. In [2] Bogaevskii constructed a different geometric framework of the Cauchy problem in the case for the Hamiltonian $\frac{1}{2} \sum_{i=1}^{n}\left(\frac{\partial y}{\partial x_{i}}\right)^{2}$. This first attempt to study of this subject, which also contains some results about the realization of singularities, was based on the theory of singularities of minimum functions. Thanks to an existence theorem for global generating families quadratic at infinity (due to Sikorav and Chekanov), the existence theorem for a global Lipshitz-continuous solution has been established by (J. C. Sikorav, C. Viterbo[16], and M. Chaperon [4,5]). This solution is known as the minimax solution which sometimes coincides (especially, when the Hamiltonian is uniformly convex or concave,) with the viscosity solution. The singualrities of the minimax solution were studied and classified by $\mathrm{T}$. Jukowskaia $([12,13])$. Therefore, it might be said that the theory for singularities of weak solutions when the Hamiltonian is uniformly convex or concave is already established by these works. So we consider the equation with a nonconvex Hamiltonian. For example, the notion of nondegeneracy for the Hamiltonian in this paper is much weaker than the condtion that the Hamiltonian is convex or concave (cf., §3). We also mention that Bogaevskii[3] has indepnedently shown realization theorems for singularities of solutions for nonconvex Hamiltonian.

We consider the case when the Hamiltonian depends only on momenta, $H\left(\frac{\partial y}{\partial x_{1}}, \cdots, \frac{\partial y}{\partial x_{n}}\right)$, in $\S 4$. In this case we can state much stronger assertion for realizations of bifircations of singularities (cf., Proposition 4.1).

All maps considered here are differentiable of class $C^{\infty}$, unless stated otherwise.

\section{Geometric framework for Hamilton-Jacobi equations.}

In the present paper we treat Hamilton-Jacobi equations in the framework of the geometric theory of first order partial differential equations described e.g., in [10]. In this section we briefly describe the geometric 
framework and present the necessary notation.

Let $J^{1}\left(\mathbb{R}^{n}, \mathbb{R}\right)$ be the 1-jet bundle of functions of $n$-variables which may be considered as $\mathbb{R}^{2 n+1}$ with a natural coordinate system

$$
\left(x_{1}, \ldots, x_{n}, y, p_{1}, \ldots, p_{n}\right),
$$

where $\left(x_{1}, \ldots, x_{n}\right)$ is a coordinate system of $\mathbb{R}^{n}$. We also have a natural projection $\pi: J^{1}\left(\mathbb{R}^{n}, \mathbb{R}\right) \rightarrow \mathbb{R}^{n} \times \mathbb{R}$ given by $\pi(x, y, p)=(x, y)$.

An immersion germ $i:\left(L_{0}, u_{0}\right) \rightarrow J^{1}\left(\mathbb{R}^{n}, \mathbb{R}\right)$ is said to be a Legendrian immersion germ (i.e., Legendrian submanifold germ) if $\operatorname{dim} L=n$ and $i^{*} \theta=$ 0 , where $\theta=d y-\sum_{i=1}^{n} p_{i} \cdot d x_{i}$. The image of $\pi \circ i$ is called the wave front set of $i$ and it is denoted by $W(i)$.

We also consider the 1-jet bundle $J^{1}\left(\mathbb{R} \times \mathbb{R}^{n}, \mathbb{R}\right)$ and the canonical 1form $\Theta$ on that space. Let $\left(t, x_{1}, \ldots, x_{n}\right)$ be a canonical coordinate system on $\mathbb{R} \times \mathbb{R}^{n}$ and

$$
\left(t, x_{1}, \ldots, x_{n}, y, s, p_{1}, \ldots, p_{n}\right)
$$

the corresponding coordinate system on $J^{1}\left(\mathbb{R} \times \mathbb{R}^{n}, \mathbb{R}\right)$. Then, the canonical 1 -form is given by

$$
\Theta=d y-\sum_{i=1}^{n} p_{i} \cdot d x_{i}-s \cdot d t=\theta-s \cdot d t
$$

We define the natural projection $\Pi: J^{1}\left(\mathbb{R} \times \mathbb{R}^{n}, \mathbb{R}\right) \rightarrow\left(\mathbb{R} \times \mathbb{R}^{n}\right) \times \mathbb{R}$ by $\Pi(t, x, y, s, p)=(t, x, y)$. We call the above 1 -jet bundle an unfolded 1-jet bundle.

A Hamilton-Jacobi equation is defined to be a hypersurface

$$
E(H)=\left\{(t, x, y, s, p) \in J^{1}\left(\mathbb{R} \times \mathbb{R}^{n}, \mathbb{R}\right) \mid s+H(t, x, p)=0\right\}
$$

in $J^{1}\left(\mathbb{R} \times \mathbb{R}^{n}, \mathbb{R}\right)$. A geometric solution of $E(H)$ is a Legendrian submanifold $L$ in $J^{1}\left(\mathbb{R} \times \mathbb{R}^{n}, \mathbb{R}\right)$ lying in $E(H)$.

We say that a generalized Cauchy problem (GCP) (with initial condition $\left.L^{\prime}\right)$ is given for an equation $E(H)$ if there is given an $n$-dimensional submanifold $i: L^{\prime} \subset E(H)$ such that $i^{*} \Theta=0$ and $X_{H} \notin T\left(L^{\prime}\right)$ at any point of $L^{\prime}$ where $X_{H}$ is the characteristic vector field given by

$$
X_{H}=\frac{\partial}{\partial t}+\sum_{i=1}^{n} \frac{\partial H}{\partial p_{i}} \frac{\partial}{\partial x_{i}}+\left(\sum_{i=1}^{n} p_{i} \frac{\partial H}{\partial p_{i}}-H\right) \frac{\partial}{\partial y}-\frac{\partial H}{\partial t} \frac{\partial}{\partial s}-\sum_{i=1}^{n} \frac{\partial H}{\partial x_{i}} \frac{\partial}{\partial p_{i}}
$$

We have the following existence theorem: 
Theorem 1.1 (Classical existence theorem [15]). A GCP $i: L^{\prime} \subset$ $E(H)$ has a unique solution, that is, there is a Legendrian submanifold $L \subset E(H), L^{\prime} \subset L$ and any two such Legendrian submanifolds coincide in a neighbourhood of $L^{\prime}$.

In order to study $(\mathrm{P})$ we need a more restricted framework. For any $c \in(\mathbb{R}, 0)$, we define

$$
E(H)_{c}=\left\{(c, x, y,-H(c, x, p), p) \mid(x, y, p) \in J^{1}\left(\mathbb{R}^{n}, \mathbb{R}\right)\right\} .
$$

Then, $E(H)_{c}$ is a $(2 n+1)$-dimensional submanifold of $J^{1}\left(\mathbb{R} \times \mathbb{R}^{n}, \mathbb{R}\right)$ and $\Theta_{c}=\Theta \mid E(H)_{c}=d z-\sum_{i=1}^{n} p_{i} d x_{i}$ gives a contact structure on $E(H)_{c}$. We define a mapping $\iota_{c}: J^{1}\left(\mathbb{R}^{n}, \mathbb{R}\right) \rightarrow E(H)_{c}$ by $\iota_{c}(x, y, p)=$ $(c, x, y,-H(c, x, p), p)$. The mapping $\iota_{c}$ is a contact diffeomorphism and the following diagram is commutative:

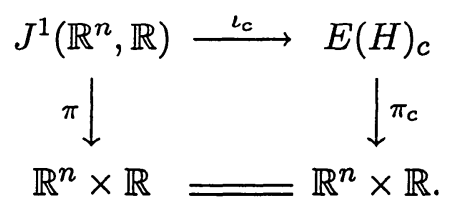

We say that a generalized Cauchy problem (with initial condition $L^{\prime}$ ) associated with the time parameter(GCPT) is given for an equation $E(H)$ if a GCP $i: L^{\prime} \subset E(H)$ with $i\left(L^{\prime}\right) \subset E(H)_{c}$ for some $c \in(\mathbb{R}, 0)$ is given.

Remark. The Cauchy problem (P) is a GCPT. The initial submanifold is given by

$$
L_{\phi, 0}=\left\{\left(0, x, \phi(x),-H\left(0, x, \frac{\partial \phi}{\partial x}\right), \frac{\partial \phi}{\partial x}\right) \mid x \in \mathbb{R}^{n}\right\} \subset E(H)_{0} .
$$

The problem of studying the singularities of the graph of the geometric solution is formulated as follows:

Geometric problem. Classify the generic bifurcations of wave fronts of

$$
\pi_{t} \mid: L \cap E(H)_{t} \rightarrow \mathbb{R}^{n} \times \mathbb{R}
$$

with respect to the parameter $t$ (i.e., the generic bifurcations of wove fronts of geometric solutions along the time parameter).

Following [10], in order to study the singularities of the geometric solution we identify geometric solutions with one-parameter Legendrian unfoldings. Such a characterization, which is given in Section 3 permits the use of 
the available singularity theory of one-parameter Legendrian unfoldings. In the next section we present the necessary background material that we use in Section 3.

\section{One parameter Legendrian unfoldings.}

We now describe the notion of one-parameter Legendrian unfoldings. Let $R$ be an $(n+1)$-dimensional smooth manifold, $\mu:\left(R, u_{0}\right) \rightarrow\left(\mathbb{R}, t_{0}\right)$ be a submersion germ and $\ell:\left(R, u_{0}\right) \rightarrow J^{1}\left(\mathbb{R}^{n}, \mathbb{R}\right)$ be a smooth map germ. We say that the pair $(\mu, \ell)$ is a Legendrian family if $\ell_{t}=\ell \mid \mu^{-1}(t)$ is a Legendrian immersion germ for any $t \in\left(\mathbb{R}, t_{0}\right)$. Then we have the following simple but very important lemma.

Lemma 2.1. Let $(\mu, \ell)$ be a Legendrian family. Then there exist a unique element $h \in C_{u_{0}}^{\infty}(R)$ such that $\ell^{*} \theta=h \cdot d \mu$, where $C_{u_{0}}^{\infty}(R)$ is the ring of smooth function germs at $u_{0}$.

Define a map germ $\mathcal{L}:\left(R, u_{0}\right) \rightarrow J^{1}\left(\mathbb{R} \times \mathbb{R}^{n}, \mathbb{R}\right)$ by

$$
\mathcal{L}(u)=(\mu(u), x \circ \ell(u), y \circ \ell(u), h(u), p \circ \ell(u)) .
$$

We can easily show that $\mathcal{L}$ is a Legendrian immersion germ. If we fix 1 -forms $\Theta$ and $\theta$, the Legendrian immersion germ $\mathcal{L}$ is uniquely determined by the Legendrian family $(\mu, \ell)$. We call $\mathcal{L}$ a Legendrian unfolding associated with the Legendrian family $(\mu, \ell)$. In order to study bifurcations of wave fronts of Legendrian unfoldings, we introduce the following equivalence relation. Let $\mathcal{L}_{i}:\left(R, u_{i}\right) \rightarrow J^{1}\left(\mathbb{R} \times \mathbb{R}^{n}, \mathbb{R}\right)(i=0,1)$ be Legendrian unfoldings. We say that $\mathcal{L}_{0}$ and $\mathcal{L}_{1}$ are $P$-Legendrian equivalent if there exist a contact diffeomorphism germ

$$
K:\left(J^{1}\left(\mathbb{R} \times \mathbb{R}^{n}, \mathbb{R}\right), z_{0}\right) \rightarrow\left(J^{1}\left(\mathbb{R} \times \mathbb{R}^{n}, \mathbb{R}\right), z_{1}\right)
$$

of the form

$$
K(t, x, y, s, p)=\left(\phi_{1}(t), \phi_{2}(t, x, y), \phi_{3}(t, x, y), \phi_{4}(t, x, y, s, p), \phi_{5}(t, x, y, s, p)\right)
$$

and a diffeomorphism germ $\Psi:\left(R, u_{o}\right) \rightarrow\left(R, u_{1}\right)$ such that $K \circ \mathcal{L}_{0}=\mathcal{L}_{1} \circ \Psi$.

In order to understand the meaning of P-Legendrian equivalence, we introduce the following equivalence: We say that two wave front sets $W\left(\mathcal{L}_{0}\right)$ and $W\left(\mathcal{L}_{1}\right)$ have diffeomorphic bifurcations if there exists a diffeomorphism germ

$$
\Phi:\left(\mathbb{R} \times\left(\mathbb{R}^{n} \times \mathbb{R}\right), \Pi\left(z_{0}\right)\right) \rightarrow\left(\mathbb{R} \times\left(\mathbb{R}^{n} \times \mathbb{R}\right), \Pi\left(z_{1}\right)\right)
$$


of the form $\Phi(t, x, y)=\left(\phi_{1}(t), \phi_{2}(t, x, y), \phi_{3}(t, x, y)\right)$ such that $\Phi(W(\mathcal{L}))=$ $W\left(\mathcal{L}^{\prime}\right)$. If $\mathcal{L}_{0}$ and $\mathcal{L}_{1}$ are P-Legendrian equivalent then these wavefronts have diffeomorphic bifurcations. By the theorem of Zakalyukin [18;Assertion, Section1.1], the converse is also true for generic Legendrian unfoldings. We can define the notion of stability with respect to the P-Legendrian equivalence in the same way as for the ordinary Legendrian stability (see [1],[18]).

Motivated by Arnol'd-Zakalyukin's theory ([1],[18]), we can construct generating families of Legendrian unfoldings. A function germ $F:((\mathbb{R} \times$ $\left.\left.\mathbb{R}^{n}\right) \times \mathbb{R}^{k}, 0\right) \rightarrow(\mathbb{R}, 0)$ is called a a generalized phase function germ if $d_{2} F \mid 0 \times$ $\mathbb{R}^{n} \times \mathbb{R}^{k}$ is non-singular, where $d_{2} F(t, x, q)=\left(\frac{\partial F}{\partial q_{1}}(t, x, q), \ldots, \frac{\partial F}{\partial q_{k}}(t, x, q)\right)$. Then $C(F)=d_{2} F^{-1}(0)$ is a smooth $(n+1)$-manifold germ and $\pi_{F}$ : $(C(F), 0) \rightarrow \mathbb{R}$ is a submersion germ, where $\pi_{F}(t, x, q)=t$.

Define map germs $\tilde{\Phi}_{F}:(C(F), 0) \rightarrow J^{1}\left(\mathbb{R}^{n}, \mathbb{R}\right)$ by

$$
\tilde{\Phi}_{F}(t, x, q)=\left(x, F(t, x, q), \frac{\partial F}{\partial x}(t, x, q)\right)
$$

and $\Phi_{F}:(C(F), 0) \rightarrow J^{1}\left(\mathbb{R} \times \mathbb{R}^{n}, \mathbb{R}\right)$ by

$$
\Phi_{F}(t, x, q)=\left(t, x, F(t, x, q), \frac{\partial F}{\partial t}(t, x, q), \frac{\partial F}{\partial x}(t, x, q)\right) .
$$

Since $\frac{\partial F}{\partial q_{i}}=0$ on $C(F)$, we can easily show that $\left(\tilde{\Phi}_{F}\right)^{*} \theta=\frac{\partial F}{\partial t}|C(F) \cdot d t| C(F)$. By definition, $\Phi_{F}$ is a Legendrian unfolding associated with the Legendrian family $\left(\pi_{F}, \tilde{\Phi}_{F}\right)$. Following the lines of Arnol'd-Zakalyukin ([1],[18]), we can show the following proposition.

Proposition 2.2. All Legendrian unfolding germs are constructed by the above method.

We define a function germ $\tilde{F}:\left(\mathbb{R} \times\left(\mathbb{R}^{n} \times \mathbb{R}\right) \times \mathbb{R}^{k}, 0\right) \rightarrow(\mathbb{R}, 0)$ by $\tilde{F}(t, x, y, q)=F(t, x, q)-y$. We call $\tilde{F}$ a generating family of $\Phi_{F}$. We also consider an equivalence relation among generating families of Legendrian unfoldings. Let

$$
\tilde{F}_{i}:\left(\mathbb{R} \times\left(\mathbb{R}^{n} \times \mathbb{R}\right) \times \mathbb{R}^{k}, 0\right) \rightarrow(\mathbb{R}, 0)(i=0,1)
$$

be generating families of $\Phi_{F_{i}}$. We say that $\tilde{F}_{0}$ and $\tilde{F}_{1}$ are $t-P-\mathcal{K}$-equivalent if there exists a diffeomorphism germ

$$
\Phi:\left(\mathbb{R} \times\left(\mathbb{R}^{n} \times \mathbb{R}\right) \times \mathbb{R}^{k}, 0\right) \rightarrow\left(\mathbb{R} \times\left(\mathbb{R}^{n} \times \mathbb{R}\right) \times \mathbb{R}^{k}, 0\right)
$$


Realization of Geometric Singularities for Hamilton-Jacobi Equations 481

of the form

$$
\Phi(t, x, y, q)=\left(\phi_{1}(t), \phi_{2}(t, x, y), \phi_{3}(t, x, y), \phi_{4}(t, x, y, q)\right)
$$

such that

$$
\left\langle\tilde{F}_{1} \circ \Phi\right\rangle_{\mathcal{E}_{(t, x, y, q)}}=\left\langle\tilde{F}_{0}\right\rangle_{\mathcal{E}_{(t, x, y, q)}},
$$

where $\left\langle\tilde{F}_{0}\right\rangle_{\mathcal{E}_{(t, x, y, q)}}$ denotes the ideal generated by $\tilde{F}_{0}$ in the ring $\mathcal{E}_{(t, x, y, q)}$ of function germs of $(t, x, y, q)$-variables at the origin. The definition of the stable $t$ - $P$ - $\mathcal{K}$-equivalence is given in the usual way (see [1],[17]).

For a generating family $\tilde{F}$ of $\Phi_{F}$, we define

$$
T_{e}(\mathrm{P}-\mathcal{K})(\tilde{f})=\left\langle\frac{\partial \tilde{f}}{\partial q_{1}}, \ldots, \frac{\partial \tilde{f}}{\partial q_{k}}, \tilde{f}\right\rangle_{\mathcal{E}_{(x, y, q)}}+\left\langle\frac{\partial \tilde{f}}{\partial x_{1}}, \ldots, \frac{\partial \tilde{f}}{\partial x_{n}}, 1\right\rangle_{\mathcal{E}_{(x, y)}}
$$

and P-K- $\operatorname{cod} \tilde{f}=\operatorname{dim}_{\mathbb{R}} \mathcal{E}_{(x, y, q)} / T_{e}(\mathrm{P}-\mathcal{K})(\tilde{f})$, where $\tilde{f}=\tilde{F} \mid 0 \times\left(\mathbb{R}^{n} \times \mathbb{R}\right) \times \mathbb{R}^{k}$. We also say that $\tilde{F}$ is a P-K -versal deformation of $\tilde{f}$ if

$$
\mathcal{E}_{(x, y, q)}=\left\langle\frac{\partial \tilde{F}}{\partial t} \mid 0 \times\left(\mathbb{R}^{n} \times \mathbb{R}\right) \times \mathbb{R}^{k}\right\rangle_{\mathbb{R}}+T_{e}(\mathrm{P}-\mathcal{K})(\tilde{f}) .
$$

Then we have the following proposition whose proof is like that of the ordinary theory of Legendrian singularities $([1],[18])$.

Proposition 2.3. (1) Let $\tilde{F}_{i}(i=0,1)$ be generating families of $\Phi_{F_{i}}$. Then $\Phi_{F_{0}}$ and $\Phi_{F_{1}}$ are P-Legendrian equivalent if and only if $\tilde{F}_{0}$ and $\tilde{F}_{1}$ are stably $t-P-\mathcal{K}$-equivalent.

(2) Let $\tilde{F}$ be a generating family of $\Phi_{F}$, then $\Phi_{F}$ is stable with respect to the $P$-Legendrian equivalence if and only if $\tilde{F}$ is a $P$-K -versal deformation of $\tilde{f}$.

We consider the Legendrian unfolding which is given by the following generating family:

$$
q_{1}^{r+1} \pm q_{2}^{2}+\sum_{i=1}^{r-1} x_{i} q^{i}-y(1 \leq r \leq n)
$$

$$
q_{1}^{2} q_{2} \pm q_{2}^{r-1}+\sum_{i=2}^{r-1} x_{i} q_{2}^{i-1}+x_{1} q_{1}-y(4 \leq r \leq n)
$$




$$
q_{1}^{r+1} \pm q_{2}^{2}+q_{1}^{r-1}\left(t \pm x_{r-1}^{2} \pm \cdots \pm x_{n}^{2}\right)
$$

$$
\begin{array}{r}
+\sum_{i=1}^{r-2} x_{i} q_{1}^{i}-y(3 \leq r \leq n+2) \\
q_{1}^{2} q_{2} \pm q_{2}^{r-1}+q_{2}^{r-2}\left(t \pm x_{r-1}^{2} \pm \cdots \pm x_{n}^{2}\right)
\end{array}
$$

$$
+\sum_{i=2}^{r-2} x_{i} q_{2}^{i-1}+x_{1} q_{1}-y(4 \leq r \leq n+2)
$$

Each germ in the above list is a generating family of P-Legendrian stable Legendrian unfoldings. Furthermore, it is known that this gives a generic classification of Legendrian unfoldings for $n \leq 5$ (cf. [11],[18]).

\section{Realization theorems.}

In this section we identify the geometric solution of a (GCP.T) introduced in Section 2 with the notion of one-parameter Legendrian unfoldings. Let $i: L^{\prime} \subset E(H)_{0} \subset E(H)$ be the initial condition of a (GCPT) and let $L$ be the unique solution. Since $X_{H} \notin T E(H)_{c}$, then $L$ is transiverse to $E(H)_{c}$ in $E(H)$ for any $c \in(\mathbb{R}, 0)$. It follows that $L_{c}=L \cap E(H I)_{c}$ is an $n$-dimensional submanifold of $E(H)_{c}$ and it satisfies $\Theta_{c} \mid L_{c}=0$ (i.e., $L_{c}$ is a Legendrian submanifold of $\left.E(H)_{c}\right)$. If we consider the local parametrization of $L$, we may assume that $L$ is the image of an immersion germ $\mathcal{L}:\left(\mathbb{R} \times \mathbb{R}^{n}, 0\right) \rightarrow E(H)$ such that $\mathcal{L} \mid\left(c \times \mathbb{R}^{n}\right)$ is a Legendrian immersion germ of $E(H)_{c}$. Hence the coordinate representation of $\mathcal{L}$ is given by $\mathcal{L}(t, u)=(t, x(t, u), y(t, u),-H(t, x(t, u), p(t, u)), p(t, u))$.

Let $\tilde{\pi}: J^{1}\left(\mathbb{R} \times \mathbb{R}^{n}, \mathbb{R}\right) \rightarrow J^{1}\left(\mathbb{R}^{n}, \mathbb{R}\right)$ be the canonical projection. defined by $\tilde{\pi}(t, x, y, s, p)=(x, y, p)$. Then the map germ $\ell=\tilde{\pi} \circ \mathcal{L}$ satisifies that $\ell_{t}=\ell \mid \pi_{1}^{-1}(t)$ is a Legendrian immersion germ for any $t \in(\mathbb{R}, 0)$. Hence $\mathcal{L}$ is a Legendrian unfolding associated with $\left(\pi_{1}, \tilde{\pi} \circ \mathcal{L}\right)$, where $\pi_{1}$ is the canonical projection $\pi_{1}:\left(\mathbb{R} \times \mathbb{R}^{n}, 0\right) \rightarrow(\mathbb{R}, 0)$. This completes the proof of the first part of Theorem 3.1. The proof of the second part is given in [10].

Theorem 3.1. (1) The local solution of the generalized Cauchy problem associated with the time parameter for the Hamilton-Jacobi equation

$$
s+H(t, x, p)=0
$$

is a Legendrian unfolding

$$
\mathcal{L}:\left(\mathbb{R} \times \mathbb{R}^{n}, 0\right) \rightarrow J^{1}\left(\mathbb{R} \times \mathbb{R}^{n}, \mathbb{R}\right)
$$


Realization of Geometric Singularities for Hamilton-Jacobi Equations 483

(2) Let

$$
\mathcal{L}:\left(\mathbb{R} \times \mathbb{R}^{n}, 0\right) \rightarrow J^{1}\left(\mathbb{R} \times \mathbb{R}^{n}, \mathbb{R}\right)
$$

be a Legendrian unfolding associated with $\left(\pi_{1}, \ell\right)$. Then there exists a $C^{\infty}$-function germ $H\left(t, x_{1}, \ldots, x_{n}, p_{1}, \ldots, p_{n}\right)$ such that $\mathcal{L}$ is a local solution of the generalized Cauchy problem associated with the time parameter for Hamilton-Jacobi equation

$$
s+H(t, x, p)=0,
$$

where the initial condition is given by $\ell(0, u)$.

The above theorem guarantees that the class of Legendrian unfoldings supplies the correct class to describe the geometric solutions of (GCPT) for Hamilton-Jacobi equations. Thus, generic results for the singularities of Legendrian unfoldings can be translated to generic results in the class of all Hamiltonians and all initial conditions. However, we must also concern ourselves with what are the types of singularities that the geometric solution to a given Hamilton-Jacobi equation might exhibit. For the purpose, we need a kind of non-degeneracy condition on the Hamiltonian function. We say that a Hamilitonian function $H(t, x, p)$ is $m$-non-degenerated at $\left(t_{0}, x_{0}, p_{0}\right)$ if $\frac{\partial^{s} H}{\partial p_{1}^{i_{1}} \ldots \partial p_{n}^{i_{n}}}\left(t_{0}, x_{0}, p_{0}\right) \neq 0$ for some $\left(i_{1}, \ldots, i_{n}\right) \in(\mathbb{N} \cup\{0\})^{n}$ with $i_{1}+\cdots+i_{n}=$ $m \geq 1$. We simply say that $H(t, x, p)$ is non-degenerated at $\left(t_{0}, x_{0}, p_{0}\right)$ if it is 2-non-degenerated at $\left(t_{0}, x_{0}, p_{0}\right)$. The following theorem is a realization theorem for generic singularities for a given Hamilton-Jacobi equation.

Theorem 3.2. Let $H(t, x, p)$ be a non-degenerate Hamiltonian function germ at $\left(t_{0}, x_{0}, p_{0}\right)$ and $\mathcal{L}:\left(R, u_{0}\right) \rightarrow\left(J^{1}\left(\mathbb{R} \times \mathbb{R}^{n}, \mathbb{R}\right),\left(t_{0}, x_{0}, y_{0}, s_{0}, p_{0}\right)\right)$ be a P-Legendrian stable Legendrian unfolding associated with $(\mu, \ell)$. Then there exists a Legendrian unfolding $\mathcal{L}^{\prime}$ which is a geometric solution of the Hamilton-Jacobi equation $s+H(t, x, p)=0$ such that $\mathcal{L}$ and $\mathcal{L}^{\prime}$ are $P$ Legendrian equivalent.

Proof. Without loss of generality, we assume that $\left(t_{0}, x_{0}, y_{0}\right)=(0,0,0)$. Let $\tilde{G}:\left(\mathbb{R} \times\left(\mathbb{R}^{n} \times \mathbb{R}\right) \times \mathbb{R}^{k}, 0\right) \rightarrow(\mathbb{R}, 0)$ be a generating family of the Legendrian unfolding $\mathcal{L}$. Since $d_{2} G \mid 0 \times \mathbb{R}^{n} \times \mathbb{R}^{k}$ is non-singular, the set

$$
\phi_{g}=\left\{\left(0, x,-H\left(0, x, \frac{\partial g}{\partial x}(x, q)\right), \frac{\partial g}{\partial x}(x, q)\right) \mid \frac{\partial g}{\partial q_{i}}(x, q)=0 i=1, \ldots, k\right\}
$$

is the initial condition for the corresponding (GCPT), where $g=G \mid 0 \times \mathbb{R}^{n} \times$ $\mathbb{R}^{k}$. By the arguments of the last part of $\S 1$, we can construct a Legendrian unfolding $\mathcal{L}^{\prime}$ which is the local unique geometric solution around $\phi_{g}$. 
We now choose a generating family $\tilde{F}:\left(\mathbb{R} \times\left(\mathbb{R}^{n} \times \mathbb{R}\right) \times \mathbb{R}^{k^{\prime}}, 0\right) \rightarrow(\mathbb{R}, 0)$ of $\mathcal{L}^{\prime}$. By definition, Image $\Phi_{F} \mid t=0$ is equal to $\phi_{g}$, so that we may assume that $k=k^{\prime}$ and $\tilde{f}, \tilde{g}$ are $P$ - $\mathcal{K}$-equivalent, where $f=F \mid 0 \times \mathbb{R}^{n} \times \mathbb{R}^{k}, \tilde{f}(x, y, q)=$ $f(x, q)-y$ and $\tilde{g}(x, y, q)=g(x, q)-y$.

If $P-\mathcal{K}$ - $\operatorname{cod} \tilde{g}=0$, then $P-\mathcal{K}$-cod $\tilde{f}=0$, so that $\tilde{f}$ is already a $P$ - $\mathcal{K}$-versal deformation of itself. Thus, $\tilde{F}$ and $\tilde{G}$ are $P-\mathcal{K}$-versal deformations of $\tilde{f}$ and $\tilde{g}$ respectively. By the uniqueness theorem of $P$ - $\mathcal{K}$-versal deformations (see $[8]), \tilde{F}$ is t- $P$ - $\mathcal{K}$-equivalent to $\tilde{G}$.

We now assume that $P-\mathcal{K}$-cod $\tilde{g}=1$, so that $P$ - $\mathcal{K}$-cod $\tilde{f}=1$. If $\left.\frac{\partial F}{\partial t}\right|_{t=0} \notin$ $T_{e}(P-\mathcal{K})(\tilde{f})$, then we can get the required assertion by the uniqueness of the $P$ - $\mathcal{K}$-versal deformation as in the previous case.

Suppose that $\left.\frac{\partial F}{\partial t}\right|_{t=0} \in T_{e}(P-\mathcal{K})(\tilde{f})$ for any generating family $\tilde{F}$ of $\mathcal{L}^{\prime}$. Since $\Phi_{F}$ is a geometric solution of $s+H(t, x, p)=0$, we have a relation

$$
-\frac{\partial F}{\partial t} \equiv H\left(t, x, \frac{\partial F}{\partial x}\right) \bmod \left\langle\frac{\partial F}{\partial q_{1}}, \ldots, \frac{\partial F}{\partial q_{k}}\right\rangle_{\mathcal{E}_{(t, x, q)}},
$$

so that

$$
-\left.\frac{\partial F}{\partial t}\right|_{t=0} \equiv H\left(0, x, \frac{\partial f}{\partial x}\right) \bmod \left\langle\frac{\partial f}{\partial q_{1}}, \ldots, \frac{\partial f}{\partial q_{k}}\right\rangle_{\mathcal{E}_{(x, q)}} .
$$

Therefore

$$
-\left.\frac{\partial F}{\partial t}\right|_{t=x=0} \equiv H\left(0,0, \frac{\partial f}{\partial x}(0, q)\right) \bmod \left\langle\frac{\partial f_{0}}{\partial q_{1}}, \ldots, \frac{\partial f_{0}}{\partial q_{k}}\right\rangle_{\mathcal{E}_{q}},
$$

where $f_{0}(q)=f(0, q)$. We may assume that $f_{0} \in \mathfrak{M}_{q}^{3}$, where $\mathfrak{M}_{q}$ is the unique maximal ideal of $\mathcal{E}_{q}$.

We now consider the Taylor polynomial of $H(t, x, p)$ of degree 2 at $\left(t, x, p_{0}\right)$ with respect to $p=\left(p_{1}, \ldots, p_{n}\right)$-variables as follows :

$$
\begin{aligned}
H(t, x, p)= & H\left(t, x, p_{0}\right)+\sum_{i=1}^{n} \frac{\partial H}{\partial p_{i}}\left(t, x, p_{0}\right)\left(p_{i}-p_{0, i}\right) \\
& +\frac{1}{2} \sum_{i, j} \frac{\partial^{2} H}{\partial p_{i} \partial p_{j}}\left(t, x, p_{0}\right)\left(p_{i}-p_{0, i}\right)\left(p_{j}-p_{0, j}\right)+\text { higher term. }
\end{aligned}
$$

If we set $t=0, x=0$, and $p_{i}=\frac{\partial f}{\partial x_{i}}(0, q)$, then

$$
\begin{aligned}
& H\left(0,0, \frac{\partial f}{\partial x_{i}}(0, q)\right)=H\left(0,0, p_{0}\right)+\sum_{i=1}^{n} \frac{\partial H}{\partial p_{i}}\left(x, p_{0}\right)\left(\frac{\partial f}{\partial x_{i}}(0, q)-p_{0, i}\right) \\
& +\frac{1}{2} \sum_{i, j} \frac{\partial^{2} H}{\partial p_{i} \partial p_{j}}\left(x, p_{0}\right)\left(\frac{\partial f}{\partial x_{i}}(0, q)-p_{0, i}\right)\left(\frac{\partial f}{\partial x_{j}}(0, q)-p_{0, j}\right)+\text { higher term. }
\end{aligned}
$$


Realization of Geometric Singularities for Hamilton-Jacobi Equations 485

Since $F(t, x, q)$ is a generalized phase function germ, $\operatorname{rank}\left(\left.\frac{\partial^{2} F}{\partial q_{i} \partial x_{j}}\right|_{t=0}\right)=$ $k$. Then we may assume that $\frac{\partial^{2} F}{\partial q_{i} \partial x_{j}}(0)=\delta_{i j}$ for $i, j=1, \ldots, k$ and $\left.\frac{\partial F}{\partial x_{\ell}}\right|_{(t, x)=(0,0)} \in \mathfrak{M}_{q}^{2}$ for $\ell=k+1, \ldots, n$. It follows that $\frac{\partial F}{\partial x_{i}}(0,0, q)-p_{0 i}=$ $q_{i}+\psi(q)$, where $\psi(q) \in \mathfrak{M}_{q}^{2}$. Since $H$ is non-degenerate, the quadratic form $\sum_{i=1}^{k} \frac{\partial^{2} F}{\partial p_{i} \partial p_{j}}\left(0,0, p_{0}\right) q_{i} q_{j}$ does not vanish.

On the other hand

$$
-\left.\frac{\partial F}{\partial t}\right|_{t=0} \in\left\langle f(x, q)-y, \frac{\partial f}{\partial q}(x, q)\right\rangle_{\mathcal{E}_{(x, y, q)}}+\left\langle 1, \frac{\partial f}{\partial x}(x, q)\right\rangle_{\mathcal{E}_{(x, y)}}
$$

It follows that

$$
H\left(0,0, \frac{\partial f}{\partial x}(0, q)\right) \in\left\langle f(x, q)-y, \frac{\partial f_{0}}{\partial q}(q)\right\rangle_{\mathcal{E}_{q}}+\left\langle 1, \frac{\partial f_{0}}{\partial x}(q)\right\rangle_{\mathbb{R}}
$$

and

$$
\begin{aligned}
& \frac{1}{2} \sum_{1 \leq i, j \leq n} \frac{\partial^{2} H}{\partial p_{i} \partial p_{j}}\left(0,0, p_{0}\right)\left(\frac{\partial f}{\partial x_{i}}(0, q)-p_{0, i}\right)\left(\frac{\partial f}{\partial x_{j}}(0, q)-p_{0, j}\right) \\
& \equiv H\left(0,0, \frac{\partial f}{\partial x}(0, q)\right)-H\left(0,0, p_{0}\right) \\
& \quad-\sum_{i=1}^{n} \frac{\partial H}{\partial p_{i}}\left(0,0, p_{0}\right)\left(\frac{\partial f}{\partial x_{i}}(0, q)-p_{0, i}\right) \bmod \mathfrak{M}_{q}^{3} \\
& =H\left(0, \frac{\partial f}{\partial x}(0, q)\right)-H\left(0,0, p_{0}\right)-\sum_{i=1}^{n} \frac{\partial H}{\partial p_{i}}\left(0,0, p_{0}\right) \frac{\partial f}{\partial x_{i}}(0, q) \\
& \quad+\sum_{i=1}^{n} p_{0, i} \frac{\partial H}{\partial p_{i}}\left(0,0, p_{0}\right) \\
& \in\left\langle 1, \frac{\partial f}{\partial x_{1}}(0, q), \ldots, \frac{\partial f}{\partial x_{n}}(0, q)\right\rangle_{\mathbb{R}}+\left\langle f_{0}, \frac{\partial f_{0}}{\partial q}\right\rangle_{\mathcal{E}_{q}} \bmod \mathfrak{M}_{q}^{3} .
\end{aligned}
$$

For any linear isomorphism $A: \mathbb{R}^{n} \rightarrow \mathbb{R}^{n}$, we have a relation

$$
\frac{\partial f(A x, q)}{\partial x_{i}}=\sum_{j=1}^{n} A_{i j} \frac{\partial f}{\partial x_{j}}(A x, q) .
$$


Since

$$
\begin{aligned}
\sum_{1 \leq i, j \leq n} \frac{\partial^{2} H}{\partial p_{i} \partial p_{j}}\left(0,0, p_{0}\right)\left(\frac{\partial f}{\partial x_{i}}(0, q)\right. & \left.-p_{0, i}\right)\left(\frac{\partial f}{\partial x_{j}}(0, q)-p_{0, j}\right) \\
& \equiv \sum_{1 \leq i, j \leq k} \frac{\partial^{2} H}{\partial p_{i} \partial p_{j}}\left(0,0, p_{0}\right) q_{i} q_{j} \bmod \mathfrak{M}_{q}^{3}
\end{aligned}
$$

and the vector space

$$
\left\langle f_{0}, \frac{\partial f_{0}}{\partial q}\right\rangle_{\mathcal{E}_{q}}+\left\langle 1, \frac{\partial f}{\partial x_{1}}(0, q), \ldots, \frac{\partial f}{\partial x_{1}}(0, q)\right\rangle_{\mathbb{R}} \bmod \mathfrak{M}_{q}^{3}
$$

is invariant under the action of the linear isomorphism $A$, then any quadratic form of $q=\left(q_{1}, \ldots, q_{k}\right)$-variables is contained in the above vector space. If there exists a quadratic form of $q$-variables that it is contained in $\left\langle f_{0}, \frac{\partial f_{0}}{\partial q}\right\rangle_{\mathcal{E}_{q}} \bmod \mathfrak{M}_{q}^{3}$, then all quadratic forms are contained in it for the same reason as above. In this case, since the vector space $\left\langle f_{0}, \frac{\partial f_{0}}{\partial q}\right\rangle_{\mathcal{E}_{q}} \bmod \mathfrak{M}_{q}^{3}$ has at most dimension $k$, then $k$ should be 1 and $f_{0}$ is an $A_{2}$-type function germ. It follows from the classification (cf. the list after Proposition 2.3) that $F(t, x, q)$ is of ${ }^{0} A_{2}$-type, so that this case is contained in the case of $P-\mathcal{K}-\operatorname{cod}(\tilde{f})=0$.

We may assume that any quadratic form of $q$-variables is contained in

$$
\left\langle 1, \frac{\partial f}{\partial x_{1}}(0, q), \ldots, \frac{\partial f}{\partial x_{n}}(0, q)\right\rangle_{\mathbb{R}} \bmod \mathfrak{M}_{q}^{3} .
$$

Since $\mathcal{K}$-cod $\left(f_{0}\right)$ is finite (for the definition of $\mathcal{K}$-finiteness, see [17]), then there exists $r \in \mathbb{N}$ such that $\mathfrak{M}_{q}^{r} \subset\left\langle f_{0}, \frac{\partial f_{0}}{\partial q}\right\rangle_{\mathcal{E}_{q}}$. By the same arguments as those of the previous paragraph, we can assert that every monomial of $q$-variables of degree 3 is contained in the vector space

$$
\left\langle 1, \frac{\partial f}{\partial x_{1}}(0, q), \ldots, \frac{\partial f}{\partial x_{n}}(0, q)\right\rangle_{\mathbb{R}}+\left\langle f_{0}, \frac{\partial f_{0}}{\partial q}\right\rangle_{\mathcal{E}_{q}} \bmod \mathfrak{M}_{q}^{4} .
$$

If there exists a monomial of degree 3 which is contained in

$$
\left\langle f_{0}, \frac{\partial f_{0}}{\partial q}\right\rangle_{\mathcal{E}_{q}} \bmod \mathfrak{M}_{q}^{4}
$$

then $k$ should be 1 and $f_{0}$ is an $A_{3}$-type function germ. It follows that

$$
\operatorname{dim}_{\mathbb{R}}\left\langle 1, \frac{\partial f}{\partial x_{1}}(0, q), \ldots, \frac{\partial f}{\partial x_{n}}(0, q)\right\rangle_{\mathbb{R}} \geq 3 \bmod \mathfrak{M}_{q}^{4} .
$$


By the clasification (cf. the list after Proposition 2.3, $F(t, x, q)$ should be of ${ }^{0} A_{3}$-type, then this case is contained in the case of $P-\mathcal{K}$-cod $(\tilde{f})=0$.

.For ${ }^{0} A_{\ell}$ or ${ }^{1} A_{\ell}$-type germs, we get same normal forms as in the list after Proposition 2.3 without the assumption $n \leq 5$ (cf. Theorem 2.2 in [18]). We can continue this procedure up to degree $r-1$. Eventually, it remains the case that every polynomial of degree $r-1$ is contained in the vector space

$$
\left\langle 1, \frac{\partial f}{\partial x_{1}}(0, q), \ldots, \frac{\partial f}{\partial x_{n}}(0, q)\right\rangle_{\mathbb{R}}+\left\langle f_{0}, \frac{\partial f_{0}}{\partial q}\right\rangle_{\mathcal{E}_{q}} \bmod \mathfrak{M}_{q}^{r}
$$

Since $\mathfrak{M}_{q}^{r} \subset\left\langle f_{0}, \frac{\partial f_{0}}{\partial q}\right\rangle_{\mathcal{E}_{q}}$, then

$$
\mathcal{E}_{q}=\left\langle 1, \frac{\partial f}{\partial x_{1}}(0, q), \ldots, \frac{\partial f}{\partial x_{n}}(0, q)\right\rangle_{\mathbb{R}}+\left\langle f_{0}, \frac{\partial f_{0}}{\partial q}\right\rangle_{\mathcal{E}_{q}}
$$

It follows that

$$
\mathcal{E}_{(x, q)}=T_{e}(P-\mathcal{K})(\tilde{f})+\mathfrak{M}_{q} \mathcal{E}_{(x, q)},
$$

so that we have $\mathcal{E}_{(x, q)}=T_{e}(P-\mathcal{K})(f)$ by the Malgrange preparation theorem. This contradicts the fact that $P-\mathcal{K}$-cod $(\tilde{f})=1$. This completes the proof.

In the list of generating families for Legendrian unfoldings in Section 2, the germs of ${ }^{1} A_{r},{ }^{1} D_{r}$ and ${ }^{1} E_{6}$ describe the situation how geometric singularities bifurcate. Especially, ${ }^{1} A_{3}$ singularity describes how the singularity appears from a smooth solution. All of these are P-Legendrian stable Legendrian unfoldings, so that these can be realized as geometric solutions at the non-degenerated point for a given Hamilton-Jacobi equation. We consider that at where such bifurcations should be appeared.

Theorem 3.3. Let $s+H(t, x, p)=0$ be a Hamilton-Jacobi equation. If one of ${ }^{1} A_{r},{ }^{1} D_{r}$ or ${ }^{1} E_{6}$ appears at a point $\left(t_{0}, x_{0}, p_{0}\right)$ of a geometric solution of $E(H)$, then $H$ is $m$-non-degenerated at $\left(t_{0}, x_{0}, p_{0}\right)$ for some $m=1, \ldots, r-1$ (for ${ }^{1} E_{6}$ case, $1 \leq s \leq 5$ ).

Before we give the proof of Teorem 3.3, we remark that the framework we condier in this paper can be generalized to the case when the Hamiltonian $H$ also depends on the $y$-variable under a suitable assumption. In this case 
the characteristic vector field is given by

$$
\begin{aligned}
X_{H}= & \frac{\partial}{\partial t}+\sum_{i=1}^{n} \frac{\partial H}{\partial p_{i}} \frac{\partial}{\partial x_{i}}+\left(\sum_{i=1}^{n} p_{i} \frac{\partial H}{\partial p_{i}}-H\right) \frac{\partial}{\partial y} \\
& -\left(\frac{\partial H}{\partial t}+s \frac{\partial H}{\partial y}\right) \frac{\partial}{\partial s}-\sum_{i=1}^{n}\left(\frac{\partial H}{\partial x_{i}}+p_{i} \frac{\partial H}{\partial y}\right) \frac{\partial}{\partial p_{i}} .
\end{aligned}
$$

We assume the following:

$\left(^{*}\right)$ The Hamiltonian flows corresponding to $X_{H}$ exist nearby the point $\left(t_{0}, x_{0}, y_{0}, s_{0}, p_{0}\right)$.

Under this assumption, we can develope exactly the same theory near by the point as the previous one. We give the proof of Theorem 3.3 under this genralized situation for the tecnical reason. So we consider the case that the Hamiltonian $H$ depends on the $(t, x, y, p)$-variables.

Proof. For the proof, we may assume that $\left(t_{0}, x_{0}, y_{0}, s_{0}, p_{0}\right)=(0,0,0,0,0)$.

We remark that for any function germ $f:\left(\mathbb{R}^{n} \times \mathbb{R}^{k}, 0\right) \rightarrow(\mathbb{R}, 0)$ with a property that $\frac{\partial f}{\partial q}(0)=\left(\frac{\partial f}{\partial q_{1}}(0), \ldots, \frac{\partial f}{\partial q_{k}}(0)\right)$ is non zero vector; we can construct a Legendrian submanifold

$$
\phi_{f}=\left\{x, f(x, q), \frac{\partial f}{\partial x}(x, q) \mid \frac{\partial f}{\partial q}(x, q)=0\right\}
$$

of the 1-jet space $J^{1}\left(\mathbb{R}^{n}, \mathbb{R}\right)$.

Firstly we prove the assertion for the germ ${ }^{1} A_{r}$. Without the loss of generaity, we may assume that $k=1$. We consider the function germ

$$
f(x, q)=q^{r+1}+q^{r-1}\left( \pm x_{r-1}^{2} \pm \cdots \pm x_{n}^{2}\right)+\sum_{i=1}^{r-2} x_{i} q^{i}-y
$$

then $\frac{\partial f}{\partial q}=(r+1) q^{r}+(r-1) q^{r-2}\left( \pm x_{r-1}^{2} \pm \cdots \pm x_{n}^{2}\right)+\sum_{i=1}^{r-1} i x_{i} q^{i-1}$, so that $\phi_{f}$ is a Legendrian submanifold of $J^{1}\left(\mathbb{R}^{n}, \mathbb{R}\right)$ whose generating family is given by

$$
\tilde{f}(x, y, q)=f(x, q)-y=q^{r+1}+q^{r-1}\left( \pm x_{r-1}^{2} \pm \cdots \pm x_{n}^{2}\right)+\sum_{i=1}^{r-2} x_{i} q^{i}-y .
$$

Since $\ell_{0}=i_{0}\left(\phi_{f}\right)$ gives the initial condition for the (GCPT), we can get a Legendrian unfolding $(\mathcal{L}, 0) \subset E(H)$ by the method of characteristics. 
Let $\tilde{G}(t, x, y, q)=G(t, x, q)-y$ be a generating family of $(\mathcal{L}, 0)$, then $\tilde{g}(x, y, q)=g(x, q)-y=\tilde{G}(0, x, y, q)$ is a generating family of $\ell_{0}$. By the proof of Theorems 19.4 and 20.8 in [1], there exists a diffeomorphism germ $\Psi:\left(\mathbb{R}^{n} \times \mathbb{R}, 0\right) \rightarrow\left(\mathbb{R}^{n} \times \mathbb{R}, 0\right)$ of the form $\Psi(x, q)=(x, \psi(x, q))$ such that $g \circ \Psi(x, q)=f(x, q)$. Define a function germ $F:\left(\mathbb{R} \times \mathbb{R}^{n} \times \mathbb{R}, 0\right) \rightarrow(\mathbb{R}, 0)$ by $F(t, x, q)=G(t, x, \psi(x, q))$, then the Legendrian unfolding Image $\Phi_{F}$ is equal to Image $\Phi_{G}=\mathcal{L}$ and $\left.F\right|_{t=0}=f$. It follows that $\Phi_{F}$ is the geometric solution of $E(H)$ and $\frac{\partial F}{\partial t}+H\left(t, x, y, \frac{\partial F}{\partial x}\right) \equiv 0 \bmod \left\langle\left.\frac{\partial F}{\partial q}\right|_{t=0}\right\rangle_{\mathcal{E}_{(t, x, y, q)}}$. If we put $t=0$, then we have

$$
\begin{aligned}
& \left.\frac{\partial F}{\partial t}\right|_{t=0}+H(0, x, y, q) \equiv 0 \\
& \bmod \left\langle(r+1) q^{r}+(r-1) q^{r-2}\left( \pm x_{r-1}^{2} \pm \cdots \pm x_{n}^{2}\right)+\sum_{i=1}^{r-2} i x_{i} q^{i-1}\right\rangle_{\mathcal{E}_{(x, y, q)}}
\end{aligned}
$$

We now consider the Taylor polynomial of $H(t, x, y, p)$ for a sufficiently higher degree at $\left(t, x, y, p_{0}\right)$ with respect to $p=\left(p_{1}, \ldots, p_{n}\right)$-variables as follows :

$$
\begin{aligned}
H(t, x, y, p)= & H(t, x, y, 0)+\sum_{i=1}^{n} \frac{\partial H}{\partial p_{i}}(t, x, y, 0) p_{i} \\
& +\frac{1}{2} \sum_{1 \leq i, j \leq n} \frac{\partial^{2} H}{\partial p_{i} \partial p_{j}}(t, x, y, 0) p_{i} p_{j}+\text { higher. }
\end{aligned}
$$

On the other hand, we have

$$
\frac{\partial f}{\partial x_{i}}= \begin{cases}q^{i} & 1 \leq i \leq r-2 \\ \pm 2 x_{i} q^{r-1} & r-1 \leq i \leq n .\end{cases}
$$

If we set $t=0$ and $p_{i}=\frac{\partial f}{\partial x_{i}}(0, q)$, then

$$
\begin{aligned}
H\left(0, x, y, \frac{\partial f}{\partial x}(0, q)\right)= & H(0, x, y, 0)+\sum_{i=1}^{r-2} \frac{\partial H}{\partial p_{i}}(0, x, y, 0) q^{i} \\
& \left.+\sum_{i=r-1}^{n} \frac{\partial H}{\partial p_{i}}(0, x, y, 0)\left( \pm 2 x_{i} q^{r-1}\right)+\Psi(0, x, y, 0)\right) q^{r-1} \\
& +\frac{1}{2} \sum_{i+j \neq r-1} \frac{\partial^{2} H}{\partial p_{i} \partial p_{j}}(0, x, y, 0) q^{i+j}
\end{aligned}
$$




$$
+\frac{1}{2} \sum_{i, j} \frac{\partial^{2} H}{\partial p_{i} \partial p_{j}}(0, x, y, 0) q^{i-1}\left( \pm 2 q^{r-1} x_{j}\right)
$$

+ higher term,

where

$$
\begin{aligned}
\Psi(0, x, y, 0)= & \frac{1}{2} \sum_{i+j=r-1} \frac{\partial^{2} H}{\partial p_{i} \partial p_{j}}(0, x, y, 0) \\
& +\frac{1}{3 !} \sum_{i+j+k=r-1} \frac{\partial^{3} H}{\partial p_{i} \partial p_{j} \partial p_{k}}(0, x, y, 0) \\
& +\cdots+\frac{1}{s !} \sum_{i_{1}+\cdots i_{s}=r-1} \frac{\partial^{s} H}{\partial p_{1}^{i_{1}} \cdots \partial p_{s}^{i_{s}}}(0, x, y, 0) \\
& +\cdots+\frac{1}{(r-1) !} \frac{\partial^{r-1} H}{\partial p_{1}^{(r-1)}}(0, x, y, 0) .
\end{aligned}
$$

So we have

$$
\begin{aligned}
\left.\frac{\partial F}{\partial t}\right|_{t=0} & +H\left(0, x, y, \frac{\partial f}{\partial x}(0, q)\right)=\left.\frac{\partial F}{\partial t}\right|_{t=0}+H(0, x, y, 0) \\
& +\sum_{i=1}^{r-1} \frac{\partial H}{\partial p_{i}}(0, x, y, 0) q^{i-1}+\sum_{i=1}^{r-1} \frac{\partial H}{\partial p_{i}}(0, x, y, 0)\left( \pm 2 x_{i} q^{r-1}\right) \\
& +\Psi(0, x, y, 0) q^{r-1}+\frac{1}{2} \sum_{i+j \neq r-1} \frac{\partial^{2} H}{\partial p_{i} \partial p_{j}}(0, x, y, 0) q^{i+j} \\
& +\frac{1}{2} \sum_{i, j} \frac{\partial^{2} H}{\partial p_{i} \partial p_{j}}(0, x, 0) q^{i-1}\left( \pm 2 q^{r-1} x_{j}\right)+\text { higher term } \\
& \equiv 0 \\
\bmod & \left\langle(r+1) q^{r}+(r-1) q^{r-2}\left( \pm x_{r}^{2} \pm \cdots \pm x_{n}^{2}\right)+\sum_{i=1}^{r-1} i x_{i} q^{i-2}\right\rangle_{\mathcal{E}_{(x, y, q)}}
\end{aligned}
$$

Since $T_{e}(\mathrm{P}-\mathcal{K})(\tilde{f})$ is equal to

$$
\begin{gathered}
\left\langle q^{r+1}+q^{r-1}\left( \pm x_{r-1}^{2} \pm \cdots \pm x_{n}^{2}\right)+\sum_{i=1}^{r-2} x_{i} q^{i}-y\right. \\
\left.(r+1) q^{r}+(r-1) q^{r-2}\left( \pm x_{r-1}^{2} \pm \cdots \pm x_{n}^{2}\right)+\sum_{i=1}^{r-2} i x_{i} q^{i-1}\right\rangle_{\mathcal{E}_{(x, y, q)}} \\
+\left\langle 1, q, \ldots, q^{r-2}, 2 q^{r-1} x_{r-1}, \ldots, 2 q^{r-1} x_{n}\right\rangle_{\mathcal{E}_{(x, y)}}
\end{gathered}
$$


we can show that every element of $\mathcal{E}_{(x, y, q)}$ except $q^{r-1}$ is contained in $T_{e}(P-\mathcal{K})(f)$. It follows that

$$
\left.\frac{\partial F}{\partial t}\right|_{t=0}+\Psi(0, x, y, 0) q^{r-1} \in T_{e}(\mathrm{P}-\mathcal{K})(\tilde{f}) .
$$

Suppose that $\frac{\partial^{m} H}{\partial p_{1}^{i_{1} \ldots \partial p_{n}^{i_{n}}}}(0,0,0,0)=0$ for $m=1, \ldots, r-1$, then $\Psi(0, x, y, 0) \in$ $\mathfrak{M}_{(x, y)}$, so that

$$
\Psi(0, x, y, 0) q^{r-1} \in T_{e}(\mathrm{P}-\mathcal{K})(\tilde{f}) .
$$

It follows that $\left.\frac{\partial F}{\partial t}\right|_{t=0} \in T_{e}(\mathrm{P}-\mathcal{K})(\tilde{f})$. This formula shows that $\tilde{F}$ cannot be a $P$ - $\mathcal{K}$-versal deformation of $\tilde{f}$. However the generating family of type ${ }^{1} A_{r}$ is a $P$ - $\mathcal{K}$-versal deformation of $\tilde{f}$, so that $\tilde{F}$ is not t- $P$ - $\mathcal{K}$-equivalent to the germ of type ${ }^{1} A_{r}$.

On the other hand, let $\tilde{F}^{\prime}$ be a generating family of a geometric solution of $s+H(t, x, y, p)=0$. Suppose that $\tilde{F}^{\prime}$ is $t$-P-K- -equivalent to the germ of

$$
F(t, x, y, q)=q^{r+1}+q^{r-1}\left(t \pm x_{r-1}^{2} \pm \cdots \pm x_{n}^{2}\right)+\sum_{i=1}^{r-2} x_{i} q^{i}(3 \leq r \leq n+2) .
$$

It follows that the germ $\tilde{f}^{\prime}=\tilde{F}^{\prime} \mid 0 \times\left(\mathbb{R}^{n} \times \mathbb{R}\right) \times \mathbb{R}$ is P-K-equivalent to $\tilde{f}=\tilde{F} \mid 0 \times\left(\mathbb{R}^{n} \times \mathbb{R}\right) \times \mathbb{R}$, so that there exists a contact diffeomorphism germ

$$
k:\left(J^{1}\left(\mathbb{R}^{n}, \mathbb{R}\right), 0\right) \rightarrow\left(J^{1}\left(\mathbb{R}^{n}, \mathbb{R}\right), 0\right)
$$

of the form

$$
k(x, y, p)=\left(\phi_{1}(x, y), \phi_{2}(x, y), \phi_{3}(x, y, p)\right)
$$

such that $k\left(\phi_{f^{\prime}}\right)=\phi_{f}$. Since $k$ is a contact diffeomorphism germ, there exists a function germ $\lambda(x, y, p)$ with $\lambda(0,0,0) \neq 0$ such that $k^{*} \theta=\lambda \cdot \theta$. We now define a diffeomorphism germ

$$
K:\left(J^{1}\left(\mathbb{R} \times \mathbb{R}^{n}, \mathbb{R}\right), 0\right) \rightarrow\left(J^{1}\left(\mathbb{R} \times \mathbb{R}^{n}, \mathbb{R}\right), 0\right)
$$

by

$$
K(t, x, y, s, p)=\left(t, \phi_{1}(x, y), \phi_{2}(x, y), \frac{1}{\lambda(x, y, p)} \cdot s, \phi_{3}(x, y, p)\right) .
$$

It is clear that $K$ is a contact diffeomorphism (i.e., $K^{*} \Theta=\lambda \cdot \Theta$ ). It follows that $K\left(E(H)_{0}\right)=E\left(\lambda \circ k^{-1} \cdot H\right)_{0}$ and $K\left(\phi_{f^{\prime}}\right)=\phi_{f}$. Since $K$ is a 
contact diffeomorphism from $\left(J^{1}\left(\mathbb{R} \times \mathbb{R}^{n}, \mathbb{R}\right), 0\right)$ onto itself, the corresponding equation $s+\lambda \circ k^{-1}(x, y, p) \cdot H(t, x, y, p)=0$ satisfies the assumption $(*)$ and $K\left(\Phi_{F}\right)$ is a geometric solution of $E\left(\lambda \circ k^{-1} \cdot H\right)$ whose initial data is given by $\phi_{f}$. Let $\tilde{G}$ be a generating family of $K\left(\Phi_{F}\right)$, then $\tilde{F}$ and $\tilde{G}$ are $t$-P- $\mathcal{K}$-equivalent by Proposition 2.3 .

On the other hand, it is easy to show that if $\frac{\partial^{m} H}{\partial p_{1}^{i_{1}} \ldots \partial p_{n}^{i_{n}}}(0,0,0,0)=0$ for $m=1, \ldots, r-1$, then $\frac{\partial^{m} \lambda \circ k^{-1} \cdot H}{\partial p_{1}^{i_{1}} \ldots \partial p_{n}^{i_{n}}}(0,0,0)=0$ for $m=1, \ldots, r-1$, By the previous arguments, $\tilde{G}$ cannot be $t$-P- $\mathcal{K}$-equivalent to $\tilde{F}$. This contradicts to the assumption that $\tilde{F}^{\prime}$ is $t$-P-K- $\mathcal{K}$-equivalent to $\tilde{F}$. Thus the Legendrian unfolding of type ${ }^{1} A_{r}$ cannot be realized as a geometric solution of $s+$ $H(t, x, y, p)=0$, where $H(t, x, y, p)$ is not non-degenerated at $(0,0,0,0)$.

Since we can prove the assertion for the germs ${ }^{1} D_{r}$ and ${ }^{1} E_{6}$ by exactly the same way as the above arguments, we omit the proof of these cases.

Corollary 3.4. If an ${ }^{1} A_{3}$ singularity appears at $\left(t_{0}, x_{0}, p_{0}\right)$, then $H(t, x, p)$ is 1- or 2-non-degenerated at $\left(t_{0}, x_{0}, p_{0}\right)$.

Example 3.5. Consider the equation : $s+p^{3}=0$ (i.e. $n=1$ and $\left.H(t, x, p)=p^{3}\right)$.

In this case the bifurcation of geometric singularities of type ${ }^{1} A_{3}: q^{4}+$ $x q+t q^{2}-y$ describe the situation how the first singularity appears from a smooth initial data. By the above theorem the generating fanily of a Legendrian unfolding which is a geometric solution of $s+p^{3}=0$ cannot be t- $P$ - $\mathcal{K}$-equivalent to the germ of type ${ }^{1} A_{3}$. Thus the Legendrian unfolding of type ${ }^{1} A_{3}$ cannot be realized as a geometric solution of $s+p^{3}=0$ at the origin.

\section{The case when the Hamiltonian depends only on momenta.}

We can assert a much stronger statement for the case that the Hamiltonian function depends only on $\left(p_{1}, \ldots, p_{n}\right)$-variables. In this case the Cauchy problem is given by

$$
\left\{\begin{array}{l}
\frac{\partial y}{\partial t}+H\left(\frac{\partial y}{\partial x_{1}}, \cdots, \frac{\partial y}{\partial x_{n}}\right)=0 \\
y\left(0, x_{1}, \cdots, x_{n}\right)=\phi\left(x_{1}, \cdots, x_{n}\right)
\end{array}\right.
$$


where $H$ and $\phi$ are $C^{\infty}$-functiions. It follows that the characteristic equation is given by

$$
\left\{\begin{array}{l}
\frac{d x_{i}}{d t}=\frac{\partial H}{\partial p_{i}}(p)(i=1, \ldots, n), \\
\frac{d p_{i}}{d t}=0(i=1, \ldots, n), \\
\frac{d y}{d t}=-H(p)+\sum_{i=1}^{n} p_{i} \cdot \frac{\partial H}{\partial p_{i}}(p), x, p \in \mathbb{R}^{n}, \\
x(0)=u, p(0)=\frac{\partial \phi}{\partial u}(u), y(0)=\phi(u), u \in \mathbb{R}^{n} .
\end{array}\right.
$$

We can explicity solve the charcteristic equation as follows:

$$
\left\{\begin{array}{l}
x_{i}(t, u)=u_{i}+t \frac{\partial H}{\partial p_{i}}\left(\frac{\partial \phi}{\partial u}(u)\right)(i=1, \ldots, n) \\
p_{i}(t, u)=\frac{\partial \phi}{\partial u_{i}}(u)(i=1, \ldots, n) \\
y(t, u)=t\left\{-H\left(\frac{\partial \phi}{\partial u}(u)\right)+\sum_{i=1}^{n} \frac{\partial \phi}{\partial u_{i}}(u) \cdot \frac{\partial H}{\partial p_{i}}\left(\frac{\partial \phi}{\partial u}(u)\right)\right\}+\phi(u)
\end{array}\right.
$$

Then we have the following proposition.

Proposition 4.1. Let $s+H(p)=0$ be a Hamilton-Jacobi equation. If a singularity of geometric solution for the Cauchy problem $\left(P^{\prime}\right)$ appears at a point $\left(t_{0}, x_{0}, p_{0}\right)$, then $H$ is non-degenrated at $\left(t_{0}, x_{0}, p_{0}\right)$.

Proof. The Jacobian matrix of the mapping $x: \mathbb{R}^{n} \rightarrow \mathbb{R}^{n}$ with respect to $u$ is

$$
\left(\begin{array}{cccc}
1+t c_{11} & t c_{12} & \ldots & t c_{1 n} \\
t c_{21} & 1+t c_{22} & \ldots & t c_{2 n} \\
\vdots & \vdots & \ddots & \vdots \\
t c_{n 1} & t c_{n 2} & \ldots & 1+t c_{n n}
\end{array}\right)
$$

where $c_{i j}=\sum_{k=1}^{n} \frac{\partial^{2} H}{\partial p_{i} \partial p_{k}}(\phi(u)) \frac{\partial^{2} \phi}{\partial u_{k} \partial u_{j}}(u)$. By the definiton of singularity of geometric solutions, the point $\left(t_{0}, x_{0}, p_{0}\right)=\left(t_{0}, x\left(t_{0}, u_{0}\right), p\left(t_{0}, u_{0}\right)\right)$ is a singularitiy of the geometric solution if and only if the rank of the Jacobian matrix of $x: \mathbb{R}^{n} \rightarrow \mathbb{R}^{n}$ is less than $n$. However, if the Hamiltonian function $H(p)$ is degenerated at the point $\left(t_{0}, x_{0}, p_{0}\right)$, then the Jacobian matrix is 
equal to the identity matrix, so that the geometric solution is non-singular at the point.

\section{References.}

[1] V.I. Arnol'd, S.M. Gusein-Zade and A.N. Varchenko, Singularities of Differentiable Maps, Volume I, Birkhauser, 1986.

[2] I.A. Bogaevskii, Modifications of singularities of minimum funitions and bifurcations of shock waves of the Burgers eqaution with vanishing viscosity, Leningrad Math. J., 1 (1990), 807-823.

[3] I.A. Bogaevskii, Perestroikas of fronts in evolutinary families, Proceedings of the Steklov Institute of Mathematics, 209 (1995), 65-83.

[4] M. Chaperon, Generating families in contact geometry and global Lipschitz-continuous solutions of Hamilton-Jacobi equations, Proceedings of the international Geometrical colloquium, Moscow 1993.

[5] M. Chaperon, On Generating Families, Andreas Floer Memorial Volume, Birkhauser 1995.

[6] M.G. Crandall and P. Lions, Viscosity solutions of Hamilton-Jacobi equations, Trans. Amer. Math. Soc. 277 (1983), 1-42.

[7] M.G. Crandall, H. Ishii and P. Lions, User's guide to viscosity solutions of second order partial differential equations, Bull. Amer. Math. Soc. $\mathbf{2 7}$ (1992), 1-67.

[8] J. Damon, The unfolding and determinacy theorems for subgroups of $\mathcal{A}$ and $\mathcal{K}$, Memoirs of Amer. Math. Soc. 50-306 (1984).

[9] S. Izumiya, The theory of Legendrian unfoldings and first order differential equations, Proceedings of the Royal Society of Edinburgh 123A (1993), 517-532.

[10] S. Izumiya, Geometric singularities for Hamilton-Jacobi equction, Advanced Studies in Pure Math. 22 (1993), 89-100.

[11] S. Izumiya and G. T. Kossioris, Semi-local classification of geometric singularities for Hamilton-Jacobi equations, Journal of Differential Equations, 118 (1995), 166-193.

[12] T.A. Jukovskaia, Metamorphoses of the Chaperon-Sikorov weak solutions of Hamilton-Jacobi equations, Proceedings of the International Geometrical colloquium, Moscow 1993. 
Realization of Geometric Singularities for Hamilton-Jacobi Equations 495

[13] T.A. Jukovskaia, Singularites de minimax et solutions faibles d'equations aux derivees partielles, these, universite Paris 7, 1994.

[14] G.T. Kossioris, Formation of singularities for viscosity solutions of Hamilton-Jacobi equations in higher dimensions, Comm. in Partial Differential Equations 18 (1993), 1085-1108.

[15] V.V. Lychagin, Local classification of non-linear first order partial differential equations, Russian Math. Surveys 30 (1975), 105-175.

[16] C. Viterbo, Generating Functions, Symplectic Geometry and Applications, Proceedings of the international Congress of Mathematics, Zurich, Birkhauser 1995.

[17] C.T.C. Wall, Finite determinacy of smooth map germs, Bull. London Math. Soc. 14 (1981), 481-539.

[18] V.M. Zakalyukin, Reconstructions of fronts and caustics depending on a parameter and versality of mappings, Journal of Soviet Math. 27 (1983), 2713-2735.

ReCeived December 7, 1995.

[February 26, 1996]

HOKKAIDO UNIVERSITY

SAPPORO 060, JAPAN

AND

UNIVERSITY OF CRETE

IRAKLION, GREECE 